\title{
Proton inelastic cross section at ultrahigh energies
}

\author{
Atri Bhattacharya $\odot,{ }^{1, *}$ Jean-René Cudell ${ }^{1, \dagger}$ Rami Oueslati ${ }^{1,+}$ and Arno Vanthieghem ${ }^{2, \S}$ \\ ${ }^{1}$ Space sciences, Technologies and Astrophysics Research (STAR) Institute, Université de Liège, \\ Bât. B5a, 4000 Liège, Belgium \\ ${ }^{2}$ High Energy Density Science Division (HEDS), SLAC National Accelerator Laboratory, \\ Menlo Park, California 94025, USA
}

(Received 16 December 2020; accepted 9 February 2021; published 16 March 2021)

\begin{abstract}
We study the consequences of high-energy collider data on the best fits to total, elastic, and inelastic cross sections for $p p$ and $p \bar{p}$ scattering using two very distinct unitarization schemes: the eikonal and the $U$-matrix. Despite their analytic differences, we find that the two schemes lead to almost identical predictions up to EeV energies, with differences only becoming significant at GUT-scale and higher energies.
\end{abstract}

DOI: 10.1103/PhysRevD.103.L051502

Man-made accelerators and indirect detection methods of high-energy cosmic rays such as extensive air showers, at the core of high-energy and multimessenger astrophysics, have drawn a particular attention to the modeling of the high-energy hadronic interactions. A comprehensive treatment of the $p p$ and $p \bar{p}$ cross sections with quantum chromodynamics being elusive for the moment, one has to rely on some generic arguments about unitarity and analyticity of the scattering matrix to derive phenomenological estimates of the high-energy total, elastic and inelastic cross sections. In that regard, experimental studies, most notably those related to cosmic-ray showers, often use the 2002 fits to the total cross section that successfully predicted the LHC $p p$ total cross section [1]. Besides the fact that there are a lot of relevant data that have since appeared [2-13], these fits have the drawback that they cannot self-consistently relate the total cross section to the elastic and inelastic ones. Since the inelastic cross section is key to computing multiple minijet production from cosmicray interactions with the atmosphere at ultrahigh energies, the relation between the total and inelastic cross sections is therefore essential to the description of extensive air showers. It is at the core of hadronic interaction models adopted in Monte Carlo event generators such as SIBYLL [14] and QGSJET [15].

\footnotetext{
*a.bhattacharya@uliege.be

jr.cudell@uliege.be

rami.oueslati@uliege.be

${ }^{\S}$ vanthieg@slac.stanford.edu
}

Published by the American Physical Society under the terms of the Creative Commons Attribution 4.0 International license. Further distribution of this work must maintain attribution to the author(s) and the published article's title, journal citation, and DOI. Funded by SCOAP.
In this letter, we want to address this problem [16]. In order to relate elastic, inelastic, and total cross sections, one needs a physics model of the elastic amplitude. This is typically made of two ingredients: an elastic amplitude at the Born level, which encapsulates the elementary exchange (and can be extracted from low-energy data), and a scheme that takes into account multiple exchanges, which become increasingly important at higher energies and without which the elastic amplitude would exceed the unitarity limit.

The Born term of interest corresponds to pomeron exchange and is reasonably constrained. We normalize the elastic amplitude $a(s, t)$ so that the differential cross section for elastic scattering is written as

$$
\frac{\mathrm{d} \sigma_{e l}}{\mathrm{~d} t}=\frac{|a(s, t)|^{2}}{16 \pi s^{2}},
$$

where $t=-q^{2}$ is the square of the momentum transfer. The Born term can then be written using the pomeron trajectory $\alpha(t)$, the proton elastic form factor $F_{1}(t)$ and the coupling pomeron-proton $g_{p}$, as

$$
a(s, t)=g_{p}^{2} F_{1}(t)^{2}\left(\frac{s}{s_{0}}\right)^{\alpha(t)} \xi(t),
$$

with $\xi(t)$ the signature factor,

$$
\xi(t)=-e^{\frac{-i \pi \alpha(t)}{2}} .
$$

The pomeron trajectory is close to a straight line [17], and we take it to be $\alpha(t)=1+\epsilon+\alpha^{\prime} t$. Nonlinearities in the trajectory for large $t$ may become consequential when considering the differential cross section $d \sigma / d|t|$, see 
Ref. [18]; however, this is beyond the scope of the current work.

At high energy, the growth of this pomeron amplitude and eventual violation of unitarity is most clearly seen in the impact-parameter representation, where the Fourier transform of the amplitude $a(s, t)$ rescaled by $2 s$ is equivalent to a partial wave,

$$
\chi(s, \boldsymbol{b})=\int \frac{\mathrm{d}^{2} \boldsymbol{q}}{(2 \pi)^{2}} \frac{a(s, t)}{2 s} \mathrm{e}^{\mathrm{i} \boldsymbol{q} \cdot \boldsymbol{b}} .
$$

The norm of the partial wave signals two important regimes. When it reaches unity, around $\sqrt{s}=2 \mathrm{TeV}$ [19], the model enters the black-disk regime-i.e., maximum inelasticity. When it reaches two, the model begins to violate unitarity. Both regimes start at small impact parameter and spread to higher values of $b$, and signal that multiple exchanges have to be taken into account [20].

It is thus necessary to introduce unitarization schemes that take into account multiple scatterings by mapping the amplitude $\chi(s, \boldsymbol{b})$ to the physical amplitude $X(s, \boldsymbol{b})$. The latter reduces to $\chi(s, \boldsymbol{b})$ for small $s$, is confined to the unitarity circle $|X(s, \boldsymbol{b})-i| \leq 1$, and bears the same relation as Eq. (4), but this time to the unitarized amplitude $A(s, t)$,

$$
X(s, \boldsymbol{b})=\int \frac{\mathrm{d}^{2} \boldsymbol{q}}{(2 \pi)^{2}} \frac{A(s, t)}{2 s} \mathrm{e}^{\mathrm{i} \boldsymbol{q} \cdot \boldsymbol{b}} .
$$

The eikonal scheme-derived for structureless bodies in optics, potential scattering, and QED-is commonly used in the literature. Another proposed scheme is the $U$-matrix scheme, which can be motivated by a form of BetheSalpeter equation [21]. Neither of these may be entirely correct in the context of QCD, but going from one to the other permits an evaluation of the systematics linked to multiple exchanges.

The eikonal scheme assumes [22]

$$
X_{E}(s, \boldsymbol{b})=\mathrm{i}\left[1-\mathrm{e}^{\mathrm{i} \chi(s, \boldsymbol{b})}\right],
$$

while the $U$-matrix scheme posits,

$$
X_{U}(s, \boldsymbol{b})=\frac{\chi(s, \boldsymbol{b})}{1-\mathrm{i} \chi(s, \boldsymbol{b}) / 2} .
$$

In terms of partial waves, the maximum inelasticity is reached in either case for $X(s, \boldsymbol{b})=\mathrm{i}$, which is also the asymptotic limit of the eikonal scheme at high $s$.

The total and elastic scattering cross sections may be readily expressed in these representations as

$$
\sigma_{\mathrm{tot}}=2 \int \mathrm{d}^{2} \boldsymbol{b} \mathfrak{\Im m}(X(s, \boldsymbol{b})), \quad \sigma_{\mathrm{el}}=\int \mathrm{d}^{2} \boldsymbol{b}|X(s, \boldsymbol{b})|^{2} .
$$

TABLE I. The values of $\chi^{2}$ resulting from independent fits to quadratic polynomials in $\log (s)$, illustrating the tensions in some parts of the dataset.

\begin{tabular}{lcr}
\hline \hline Dataset & Number of points & $\chi^{2}$ \\
\hline$\sigma_{\text {tot }}$ & 18 & 21.7 \\
$\sigma_{e l}$ & 11 & 21.3 \\
$\sigma_{\text {in }}$ & 8 & 4.1 \\
\hline \hline
\end{tabular}

We shall now use them to fit all the data in $p \stackrel{(-)}{p}$ scattering above $500 \mathrm{GeV}$, for which lower trajectories have a negligible effect. We obtain three distinct datasets (for total, elastic and inelastic cross sections) from the following sources, for a total of 37 data points:

(i) $p p$ total and elastic cross sections from TOTEM [2-6], and ATLAS $[7,8]$;

(ii) $p \bar{p}$ total and elastic cross sections from CDF [23], E710 [24,25], and E811 [26,27] experiments at TeVatron; and UA4 at Sp̄̄S [28];

(iii) Direct measurements of inelastic cross sections, i.e., not derived from total and elastic measurements, from UA5 at Spp̄S [29], ATLAS [9,10], LHCb [11], ALICE [12], and TOTEM [13].

It should be noted that both the total and elastic cross section datasets include discordant data from different experiments. This is quantified by a simple consistency check that fits generic quadratic polynomials in $\log s$ to each dataset and computes the resulting $\chi^{2}$. Table I shows the results with both the elastic and total cross sections running up $\chi^{2} /$ d.o.f noticeably greater than 1 . Thus, one obtains a minimum combined $\chi^{2}$ of 47.1. This is a wellknown problem with these data, first addressed in [30] and later in [31]. At present, however, the number of data points is simply too small to identify individual outliers, and hence there is little one can do for lack of better experimental results. We shall thus neither filter nor sieve the data, but remember that the best possible $\chi^{2}$ is rather high.

We use a dipole form factor for the proton $F_{1}=1 /$ $\left(1-t / t_{0}\right)^{2}$. The parameters in our fit thus include $\epsilon$ and $\alpha^{\prime}$ describing the pomeron trajectory, the coupling constant $g_{p}$, and finally the form-factor scale $t_{0}$.

The results of our fits using either unitarization scheme are shown in Table II and in Fig. 1. We obtain $\chi^{2} /$ d.o.f. $=$ $1.436(1.442)$ when using the $U$-matrix (eikonal) scheme. Note that, although at face value the fit obtained using either scheme only has a seemingly poor $\chi^{2} /$ d.o.f., the

TABLE II. $\chi^{2} /$ d.o.f. and best-fit parameters obtained using the eikonal and $U$-matrix unitarization schemes.

\begin{tabular}{cccccc}
\hline \hline Scheme & \multirow{2}{*}{$\epsilon$} & $\alpha^{\prime}$ & $g_{p}$ & $t_{0}$ & $\frac{\chi^{2}}{\text { d.o.f }}$ \\
\hline Eikonal & $0.11 \pm 0.01$ & $0.31 \pm 0.19$ & $7.3 \pm 0.9$ & $1.9 \pm 0.4$ & 1.442
\end{tabular}

$\begin{array}{llllll}\text { U-matrix } & 0.10 \pm 0.01 & 0.37 \pm 0.28 & 7.5 \pm 0.8 & 2.5 \pm 0.6 & 1.436\end{array}$ 


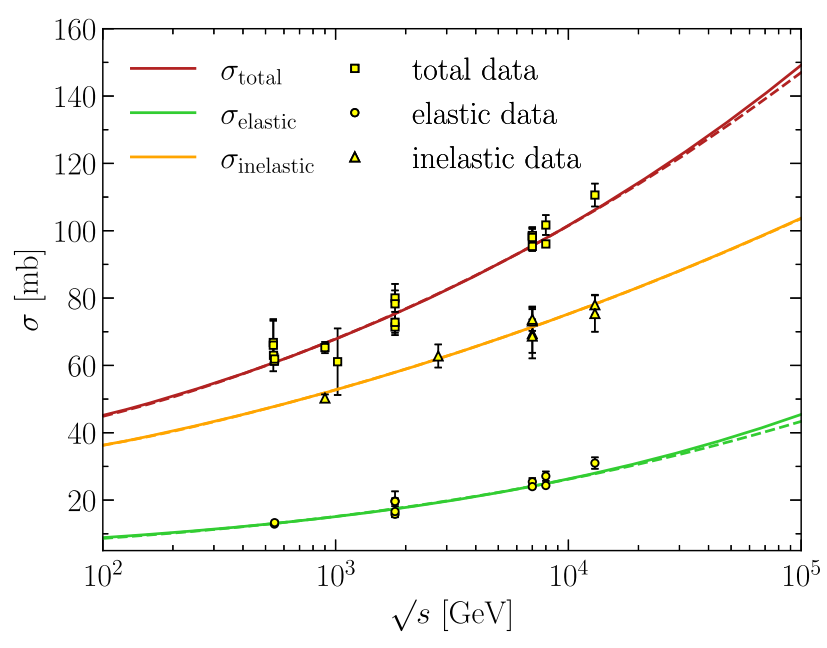

FIG. 1. Total, elastic and inelastic cross sections obtained with best-fit parameters for the $U$-matrix scheme (solid curves) and the eikonal scheme (dashed curves).

value of the total $\chi^{2}-47.39$ (47.59) for the $U$-matrix (eikonal) scheme-is very close to the minimum value47.1—obtained earlier.

These values of the parameters are however quite striking. Reference [17] managed to disentangle the pomeron contribution at low energy from that of lower- $t$ trajectories, and provided estimates of its coupling, intercept and slope. These values are within $1 \sigma$ of those obtained here for the $U$ matrix, but the eikonal differs significantly from the low-energy results. Hence it seems that for an eikonal scheme, one never recovers the observed one-pomeron simple pole.

Using an exponential form factor $\left\{F_{1}=\exp \left(R_{0} t\right)\right\}$, instead of the dipole form, leads to slightly poorer fits $\left\{\chi^{2} /\right.$ d.o.f. $\left.=1.440(1.445)\right\}$; however, the qualitative picture remains unaltered. We have also analyzed how the fits improve if one uses the generalized eikonal and $U$-matrix schemes, and we find that these generalizations - at the cost of an additional free parameter $\left(\omega\right.$ or $\left.\omega^{\prime}\right)$-do not improve the fits significantly.

One particular consequence of the relative independence of the elastic cross section to the choice of the unitarization scheme is that values of the $\rho$ parameter remain largely unaffected by the choice of the scheme as well. We use our best-fit parameters to compute this parameter across different energies, and find that the corresponding values agree with existing data, except for the latest TOTEM measurement. We indeed obtain $\rho=0.131$ at $\sqrt{s}=13 \mathrm{TeV}$. Whether this discrepancy is due to the fact that we neglect an odderon contribution, or it comes from a problem in the extraction of $\rho$ from the data [32] is still unclear. As the purpose of this paper is the evaluation of the inelastic cross section, the exact value of $\rho$ is of little importance given than it contributes about $1 \%$ to the processes considered here.

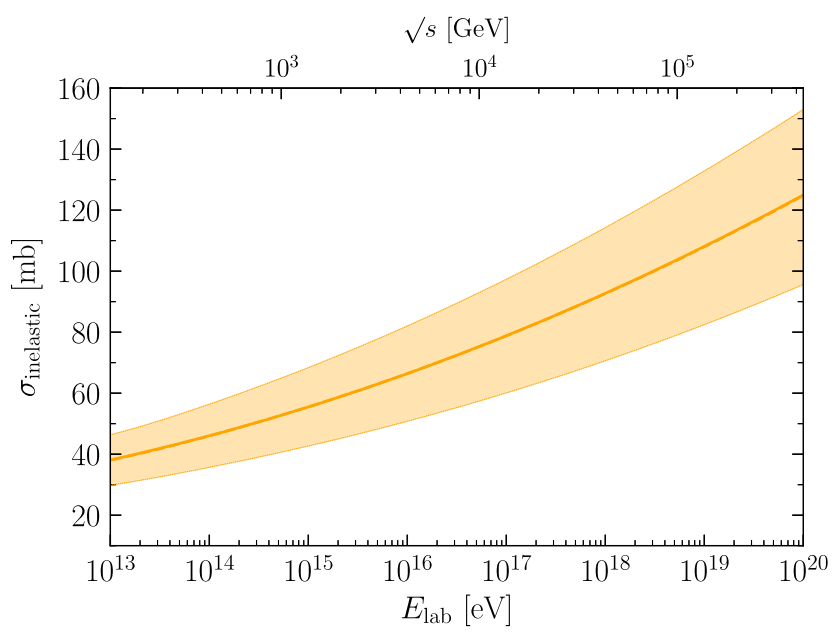

FIG. 2. The $1 \sigma$ band for the inelastic cross section at ultrahigh energies. Note that both schemes give almost identical results.

We are now in a position to present our results on the inelastic cross section at ultrahigh energies. We obtain them by varying all the parameters of Table II in a $1 \sigma$ hyperellipsoid and use the corresponding curves to evaluate the errors at ultrahigh energies. We show the results in Fig. 2. The entwinement of the inelastic cross section with the elastic and total cross sections, which are much better known, leads to smaller errors than in the case of a fit to inelastic data alone. Furthermore, despite their very different analytic properties, the two schemes lead to almost identical predictions. This gives us confidence that the extrapolation to ultrahigh energies is well founded.

While the inelastic cross sections using either of the two schemes are almost identical, this alignment happens despite significant differences in the individual order-byorder amplitudes in the expansion. We show this for the specific case of the Born term in Fig. 3. Specifically as it

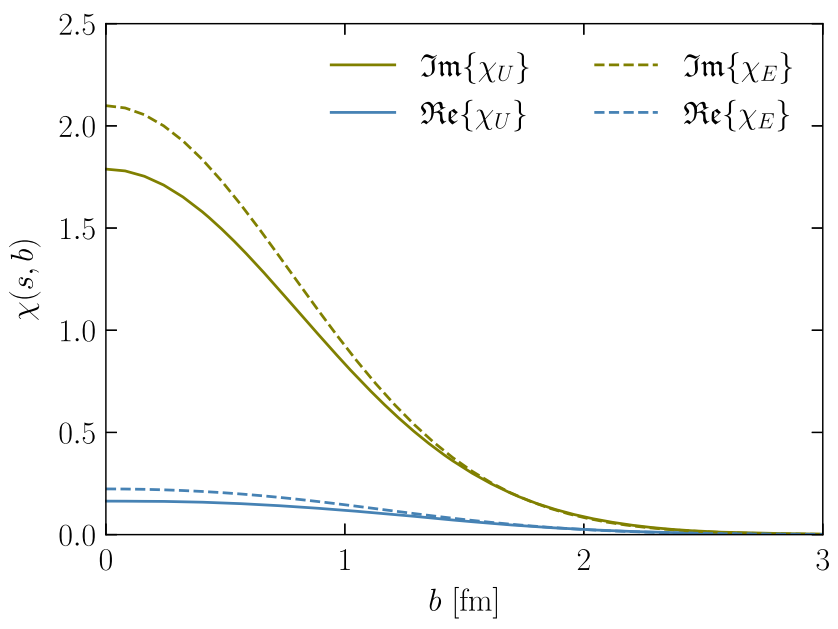

FIG. 3. Real and imaginary parts of the Born terms $\chi(s, b)$ at $\sqrt{s}=13 \mathrm{TeV}$ for the $U$-matrix (solid curves) and eikonal (dashed curves) schemes. 


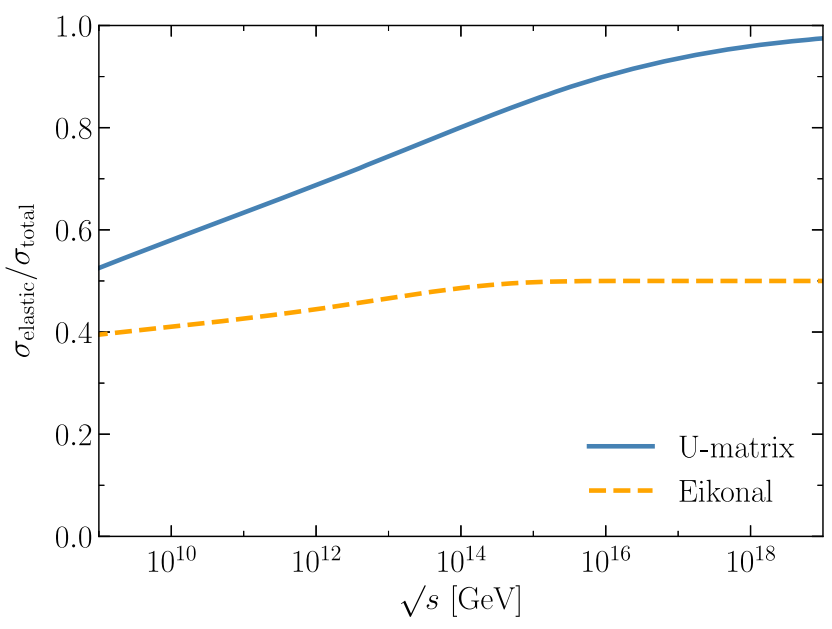

FIG. 4. The evolution of the ratio of the elastic cross section to the total cross section at the GUT-scale and higher energies based on the unitarization scheme chosen.

pertains to the inelastic cross section, this order-by-order difference can have major consequences, for example, in Monte Carlo showering codes that depend on the $n$th term in the Taylor expansion inn $\chi$ to weigh the probability of the $n$ minijets. In these codes, switching the traditionally used eikonal scheme to the U-matrix scheme will have an impact on the results, although a full analysis of this impact is beyond the scope of this work.

It is important to note that, although we have shown that the total, elastic, and inelastic cross sections obtained using the two schemes remain nearly identical for $\sqrt{s}$ up to tens of $\mathrm{TeV}$, at extremely high $\sqrt{s}$ approaching the grand unification scale the elastic and inelastic cross sections start differing significantly (see also discussion in [33]). Whereas with the eikonal scheme the elastic cross section reaches parity with the inelastic cross section at around $\sqrt{s}=10^{15} \mathrm{GeV}$ and remains so at higher energies, the $U$-matrix scheme instead predicts continuing growth for the elastic cross section-at the cost of the inelastic cross section - until it gradually approaches saturation with respect to the total cross section at some $\sqrt{s} \gtrsim 10^{19} \mathrm{GeV}$.
This is illustrated in terms of the ratio of the elastic to total cross sections in Fig. 4. These extremely high energies are of course beyond the reach of experiments; such differences are therefore of limited practical relevance.

To summarize, we have used nondiffractive experimental data from colliders up to $\sqrt{s}=13 \mathrm{TeV}$ to determine the most up-to-date fits to the total, elastic, and inelastic $p \stackrel{(-)}{p}$ cross sections in the literature, both for the eikonal and U-matrix unitarization schemes. The upshot of our analysis is that the $U$-matrix scheme leads to cross sections that fit the data as well as the eikonal scheme, which is more conventionally used in most current cosmic-ray Monte Carlo codes. The corresponding total, elastic, and inelastic cross sections from both schemes are nearly indistinguishable at energies relevant to current and nearfuture colliders; they only start showing differences at energies approaching the grand unification scale. In particular, this allows us to extrapolate the inelastic cross section up to GZK cutoff energies $\left(\sim 10^{20} \mathrm{eV}\right)$ uniquely, irrespective of the unitarization scheme chosen. This alignment between the overall inelastic cross sections notwithstanding, the amplitudes at each order in the series expansions differ significantly, with potential consequences for Monte Carlo showering codes.

\section{ACKNOWLEDGMENTS}

The authors are grateful to O. V. Selyugin and S. M. Troshin for useful discussions. A. B. is supported by the Fonds de la Recherche Scientifique-FNRS, Belgium, under Grant No. 4.4503.19. AB is thankful to the computational resource provided by Consortium des Équipements de Calcul Intensif (CÉCI), funded by the Fonds de la Recherche Scientifique de Belgique (F. R. S.-FNRS) under Grant No. 2.5020.11 where a part of the computation was carried out. This work was also supported by the Fonds de la Recherche Scientifique-FNRS, Belgium, under Grant No. 4.4503.19. A. V. is supported by U.S. DOE Early Career Research Program under Program No. FWP100331.
[1] J. R. Cudell, V. V. Ezhela, P. Gauron, K. Kang, Y. V. Kuyanov, S. B. Lugovsky, E. Martynov, B. Nicolescu, E. A. Razuvaev, and N. P. Tkachenko (COMPETE Collaboration), Phys. Rev. Lett. 89, 201801 (2002).

[2] G. Antchev et al. (TOTEM Collaboration), Europhys. Lett. 96, 21002 (2011).

[3] G. Antchev et al. (TOTEM Collaboration), Europhys. Lett. 101, 21002 (2013).

[4] G. Antchev et al. (TOTEM Collaboration), Europhys. Lett. 101, 21004 (2013).
[5] G. Antchev et al. (TOTEM Collaboration), Phys. Rev. Lett. 111, 012001 (2013).

[6] G. Antchev et al. (TOTEM Collaboration), Eur. Phys. J. C 79, 103 (2019).

[7] G. Aad et al. (ATLAS Collaboration), Nucl. Phys. B889, 486 (2014).

[8] M. Aaboud et al. (ATLAS Collaboration), Phys. Lett. B 761, 158 (2016).

[9] G. Aad et al. (ATLAS Collaboration), Nat. Commun. 2, 463 (2011). 
[10] M. Myska (ATLAS Collaboration), Proc. Sci., ICHEP2016 (2017) 1127.

[11] R. Aaij et al. (LHCb Collaboration), J. High Energy Phys. 06 (2018) 100.

[12] B. Abelev et al. (ALICE Collaboration), Eur. Phys. J. C 73, 2456 (2013).

[13] G. Antchev et al. (TOTEM Collaboration), Europhys. Lett. 101, 21003 (2013).

[14] F. Riehn, R. Engel, A. Fedynitch, T. K. Gaisser, and T. Stanev, Phys. Rev. D 102, 063002 (2020).

[15] S. Ostapchenko, EPJ Web Conf. 208, 11001 (2019).

[16] The question of the very forward component of the showers, which is linked to the diffractive cross section, will be considered in a separate paper.

[17] J. R. Cudell, A. Lengyel, and E. Martynov, Phys. Rev. D 73, 034008 (2006).

[18] L. Jenkovszky, R. Schicker, and I. Szanyi, Int. J. Mod. Phys. E 27, 1830005 (2018).

[19] J. R. Cudell and O. V. Selyugin, Czech. J. Phys. 54, A441 (2004).

[20] S. M. Troshin and N. E. Tyurin, Mod. Phys. Lett. A 31, 1650079 (2016).

[21] A. Logunov, V. Savrin, N. Tyurin, and O. Khrustalev, Teor. Mat. Fiz. 6, 157 (1971).
[22] J. R. Cudell, E. Predazzi, and O. V. Selyugin, Phys. Rev. D 79, 034033 (2009).

[23] F. Abe et al. (CDF Collaboration), Phys. Rev. D 50, 5518 (1994).

[24] N. A. Amos et al. (E-710 Collaboration), Phys. Lett. B 243, 158 (1990).

[25] N. A. Amos et al. (E710 Collaboration), Nuovo Cimento A 106, 123 (1993).

[26] C. Avila et al. (E811 Collaboration), Phys. Lett. B 445, 419 (1999).

[27] C. Avila et al. (E-811 Collaboration), Phys. Lett. B 537, 41 (2002).

[28] M. Bozzo et al. (UA4 Collaboration), Phys. Lett. 147B, 392 (1984).

[29] G. J. Alner et al. (UA5 Collaboration), Z. Phys. C 32, 153 (1986).

[30] J. R. Cudell, K. Kang, and S. K. Kim, Phys. Lett. B 395, 311 (1997).

[31] M. M. Block, Nucl. Instrum. Methods Phys. Res., Sect. A 556, 308 (2006).

[32] V. Ezhela, V. Petrov, N. Tkachenko, and A. Logunov, arXiv:2003.03817.

[33] S. M. Troshin and N. E. Tyurin, Europhys. Lett. 129, 31002 (2020). 